\title{
Levi Curvature with Radial Symmetry: a Sphere Theorem for Bounded Reinhardt Domains of $\mathrm{C}^{2}$
}

\author{
Givlio Tralli
}

ABSTRACT - We study bounded Reinhardt domains of $\mathrm{C}^{2}$, whose Levi curvature depends on the distance from the origin. We prove that a radial decrease of the Levi curvature forces the domain to be a ball.

\section{Introduction.}

The notion of Levi curvature for a real manifold was implicitly introduced by E. E. Levi in 1909 to characterize domains of holomorphy of $\mathrm{C}^{2}$. Since then, it has played a crucial role in the geometric theory of several complex variables.

Let $\partial D=\left\{z \in \mathbb{C}^{2}: F(z)=0\right\} \subset \mathbb{C}^{2}$ be a real manifold, boundary of the domain $D=\left\{z \in \mathbb{C}^{2}: F(z)<0\right\}$. We assume $F$ is a real-valued function with continuous second-order derivatives and such that

$$
\partial_{p} F=\left(\frac{\partial F}{\partial z_{1}}(p), \frac{\partial F}{\partial z_{2}}(p)\right)=\left(F_{1}(p), F_{2}(p)\right) \neq 0 \quad \forall p \in \partial D .
$$

Let us denote by $T_{p}^{\mathrm{C}}(\partial D)$ the complex tangent space to $\partial D$ at the point $p$,

$$
T_{p}^{\mathrm{C}}(\partial D)=\left\{\left(h_{1}, h_{2}\right) \in \mathbb{C}^{2}: h_{1} F_{1}(p)+h_{2} F_{2}(p)=0\right\},
$$

which is a complex line generated by the unitary vector

$$
u=\left(u_{1}, u_{2}\right)=\frac{1}{\left|\partial_{p} F\right|}\left(-F_{2}(p), F_{1}(p)\right) .
$$

Indirizzo dell'A.: Dipartimento di Matematica, Università di Bologna, Piazza di Porta S. Donato 5, 40126 Bologna, Italy.

E-mail: giulio.tralli2@unibo.it 
Using the notation $F_{j, \bar{k}}$ instead of $\frac{\partial^{2} F}{\partial z_{j} \partial \bar{z}_{k}}$, the Levi curvature of $\partial D$ at the
point $p$ is the real number

$$
K_{p}(\partial D)=\frac{1}{\left|\partial_{p} F\right|} \sum_{j, k=1}^{2} F_{j, \bar{k}} u_{j} \bar{u}_{k} .
$$

The domain $D$ is strictly Levi-pseudoconvex iff the Levi curvature is strictly positive at any point of $\partial D$. The Levi curvature is independent of the defining function $F$ (see, e.g., [5]): nevertheless, it implicitly depends on the orientation of $\partial D$. Moreover, by direct computation we obtain that

$$
K_{p}(\partial D)=-\frac{1}{\left|\partial_{p} F\right|^{3}} \operatorname{det}\left(\begin{array}{ccc}
0 & F_{\overline{1}} & F_{\overline{2}} \\
F_{1} & F_{1, \overline{1}} & F_{1, \overline{2}} \\
F_{2} & F_{2, \overline{1}} & F_{2, \overline{2}}
\end{array}\right) .
$$

It is easy to compute the Levi curvature of the sphere of radius $R$, boundary of the ball

$$
D_{R}=\left\{z \in \mathbb{C}^{2}:\left|z_{1}\right|^{2}+\left|z_{2}\right|^{2}<R^{2}\right\}
$$

We have

$$
K_{p}\left(\partial D_{R}\right)=\frac{1}{R} \quad \forall p \in \partial D_{R},
$$

that is the Levi curvature of the sphere is constant at any point. Spheres are not the only hypersurfaces with constant Levi curvature: if we consider the cylinder

$$
C_{R}=\left\{z \in \mathbb{C}^{2}:\left(\operatorname{Re}\left(z_{1}\right)\right)^{2}+\left(\operatorname{Re}\left(z_{2}\right)\right)^{2}<R^{2}\right\},
$$

we readily get

$$
K_{p}\left(\partial C_{R}\right)=\frac{1}{2 R} \quad \forall p \in \partial C_{R} .
$$

In [1] Hounie and Lanconelli proved that a bounded Reinhardt domain of $\mathrm{C}^{2}$ with $C^{2}$ boundary of constant Levi curvature is a ball. We would like to stress that $C_{R}$ is neither bounded nor Reinhardt.

The notion of Levi curvature has been extended to real hypersurfaces embedded in complex spaces of higher dimension by considering the Levi form of their defining function, which is the biholomorphic invariant part of the Hessian form (see, e.g., [3]). In analogy with the real case, in the case of $\mathbb{C}^{n+1}$ one defines $K^{(j)}$ to be the Levi curvature of order $j(1 \leq j \leq n)$ by taking the $j$ th elementary symmetric function of the eigenvalues of the Levi form: for details we refer to [5]. The Levi curvature of order $n$ (the 
product of the $n$ eigenvalues) is called the total Levi curvature: in the case $n=1$ it coincides with the definition introduced above.

The knowledge of the Levi curvature provides less geometric information than the Gauss curvature because the Levi form is only a part of the second fundamental form. Nonetheless, the problem of characterizing hypersurfaces with constant Levi curvature has been studied by many authors. In [2] Hounie and Lanconelli extended their previous result and proved that a bounded Reinhardt domain of $\mathrm{C}^{n+1}$ with $C^{2}$ boundary of constant Levi curvature and with rotational symmetry in two complementary sets of variables is a ball. In [6] Monti and Morbidelli proved that the only Levi umbilical hypersurfaces in $\mathbb{C}^{n+1}$ (for $n \geq 2$ ) with all constant Levi curvatures are spheres or cylinders. In [4] Martino and Montanari proved a "Soap Bubble Theorem" for a bounded star-shaped domain with smooth boundary: if the $j$ th Levi curvature $K^{(j)}$ is a positive constant and if the mean curvature of the boundary is bounded from above by $\left(K^{(j)}\right)^{\frac{1}{j}}$, then the domain is a ball.

In this paper, we consider a bounded Reinhardt domain containing the origin and we suppose it has a prescribed strictly positive Levi curvature depending on the distance from the origin. Under suitable and quite natural conditions on the prescribed curvature function, we prove that the domain must be a ball. In Section 2 we use the intrinsic additional symmetries of Reinhardt domains to express the condition of assigned Levi curvature by the second order singular ODE:

$$
s f f^{\prime \prime}=s f^{\prime 2}-H(s+f)\left(f+s f^{\prime 2}\right)^{\frac{3}{2}}-f f^{\prime} .
$$

In Section 3 we prove an existence and uniqueness result of solutions to (2) with a given initial value. Assuming the function $H$ in (2) is nonincreasing and using a tecnique basically introduced in [1], in the last section we prove the following theorem, which is the main result of this paper.

THEOREM 1.1. Let $D \subseteq \mathrm{C}^{2}$ be a bounded Reinhardt domain containing the origin. Let us assume that $\partial D$ is of class $C^{4}$ and it has an assigned Levi curvature given by

$$
K_{z}(\partial D)=H\left(\left|z_{1}\right|^{2}+\left|z_{2}\right|^{2}\right), \quad z \in \partial D,
$$

where $H$ is a strictly positive function defined in an open interval containing $\left[\min _{z \in \partial D}\|z\|^{2}, \max _{z \in \partial D}\|z\|^{2}\right]$. Let us suppose in addition that the function $H$ is nonincreasing. Then $\partial D$ is a sphere centered at the origin and its 
radius $r>0$ satisfies

$$
H\left(r^{2}\right)=\frac{1}{r} .
$$

Note 1.2. We would like to stress that (3) yields a necessary condition for the function $H$ to express the Levi curvature of some domain satisfying our requirements. Indeed, (3) implies the existence of a number $a>0$ such that $H(a) \sqrt{a}=1$.

\section{Levi curvature depending on the radius.}

Let us denote by $D \subset \mathbb{C}^{2}$ a bounded Reinhardt domain containing 0 and such that $\partial D$ is a real manifold of class $C^{4}$. A domain $D \subset C^{2}$ is called a Reinhardt domain (with respect to the origin) if

$$
\left(z_{1}, z_{2}\right) \in D \quad \Rightarrow \quad\left(e^{i t} z_{1}, e^{i s} z_{2}\right) \in D
$$

for all $s, t \in \mathbb{R}$. The interest in Reinhardt domains in several complex variables is related to the fact that the power series expansion about 0 of any holomorphic function converges normally on such domains (see, e.g., [3]). By the definition of Reinhardt domain, we get that $D$ is completely determined by its intersection with the real plane $\left\{\operatorname{Im} z_{1}=\operatorname{Im} z_{2}=0\right\}$. Therefore, to study $\partial D$ we consider the set

$$
C=\left\{\left(x_{1}, x_{2}\right) \in \mathbb{R}^{2}:\left(x_{1}+i 0, x_{2}+i 0\right) \in \partial D\right\},
$$

which is a planar curve of $\mathbb{R}^{2}$. Symmetry properties of Reinhardt domains force $C$ to be invariant with respect to $\left(x_{1}, x_{2}\right) \mapsto\left( \pm x_{1}, \pm x_{2}\right)$. In a neighborhood of a point $(a, 0) \in C$ (we can suppose $a>0$ because $0 \notin \partial D$ ), $C$ is thus described by the equation $x_{1}=g\left(x_{2}\right)$, where $g$ is an even function of class $C^{4}$. Let us represent the set $C$ locally as $x_{1}^{2}=f\left(x_{2}^{2}\right)$, where $f(s)=g^{2}(\sqrt{s})$. The fact that $g$ is even and the regularity of $g$ allow us to state that $f(s)=g^{2}(\sqrt{s})$ defines a $C^{2}$ function in a right neighborhood of 0 (see [1] for a proof of this fact). Therefore, in a neighborhood of $(a+i 0,0)$ the points of $\partial D$ satisfy $F\left(z_{1}, z_{2}\right):=\left|z_{1}\right|^{2}-f\left(\left|z_{2}\right|^{2}\right)=0$. If the region bounded by $C$ lies below the graph of $f, F$ is exactly the defining function of $\partial D$ (at least locally): then, because of the relation (1), the Levi curvature of $\partial D$ is given by

$$
K\left(z_{1}, z_{2}\right)=-\left.\frac{f(s)\left(f^{\prime \prime}(s) s+f^{\prime}(s)\right)-s f^{\prime}(s)^{2}}{\left(f(s)+s f^{\prime}(s)^{2}\right)^{\frac{3}{2}}}\right|_{s=\left|z_{2}\right|^{2}} .
$$


On the other hand, if the region bounded by $C$ lies above the graph of $f$, the defining function of $\partial D$ is $-F$ and the Levi curvature is given instead by

$$
K\left(z_{1}, z_{2}\right)=\left.\frac{f(s)\left(f^{\prime \prime}(s) s+f^{\prime}(s)\right)-s f^{\prime}(s)^{2}}{\left(f(s)+s f^{\prime}(s)^{2}\right)^{\frac{3}{2}}}\right|_{s=\left|z_{2}\right|^{2}} .
$$

Let us suppose that $\partial D$ has an assigned strictly positive Levi curvature which depends on the squared distance from the origin, i.e.

$$
K\left(z_{1}, z_{2}\right)=H\left(\left|z_{1}\right|^{2}+\left|z_{2}\right|^{2}\right) .
$$

Since $\partial D$ is of class $C^{4}$, the Levi curvature $K$ is a $C^{2}$ function, so that $H$ is of class $C^{2}$, too.

Let us define $t=\sup \{a>0:(a, 0) \in C\}$. Since each half line outgoing from 0 intersects $C, t$ is the supremum of a non-empty set and it is finite because of the boundedness of $D$. Let us represent $C$ just in a neighborhood of $(t, 0)$. Then, we can take $f$ as above and the region bounded by $C$ lies below the graph of $f$. Because of (4), $f$ has to satisfy the second order ODE described by (2): therefore

$$
s f f^{\prime \prime}=s f^{\prime 2}-H(s+f)\left(f+s f^{\prime 2}\right)^{\frac{3}{2}}-f f^{\prime} .
$$

After noting that $f^{\prime}(0)<0$, we stress that $f^{\prime}$ cannot vanish at a first point $s_{1}>0$ such that $f\left(s_{1}\right)>0$ : otherwise, the equation (2) would imply that $f^{\prime \prime}\left(s_{1}\right)<0$, contradicting the fact that $f$ is decreasing in $\left[0, s_{1}\right]$. Hence, there is a maximal interval $[0, S)$ in which we have $f^{\prime}(s)<0$ and $f(s)>0$ (note that $S<+\infty$ because of the boundedness of $D$ ).

REMARK 2.1. Straightforward calculations show that the inverse function of $f$ satisfies the same differential equation. This result is also a consequence of geometric arguments. In fact, the graph of the inverse function is obtained from the graph of $f$ by exchanging the coordinates, i.e. by performing a unitary transformation in $\mathrm{C}^{2} \operatorname{such}$ as $\left(z_{1}, z_{2}\right) \stackrel{A_{t, s}}{\rightarrow}\left(e^{i s} z_{2}, e^{i t} z_{1}\right)$ (for $s, t \in \mathbb{R}$ ). On the other hand, complex linear unitary transformations preserve the distance from the origin and it has been proved in [2] that they are the only biholomorphisms that also preserve the Levi curvature.

We can now prove the following proposition.

Proposition 2.2. With the previous notation, we have

$$
f(S)=0 .
$$


Proof. By the maximality of $S$, it follows that $f(S)=0$ or $f^{\prime}(S)=-\infty$. Suppose that $f(S)>0$ and $f^{\prime}(S)=-\infty$ : we will show that this leads to a contradiction. The inverse function $h$ is strictly decreasing in the interval $[f(S), f(0)]=[\alpha, \beta]$ and from our assumptions we get $h^{\prime}(\alpha)=0$. Thanks to the last remark, $h$ satisfies

$$
\sigma h h^{\prime \prime}=\sigma h^{\prime 2}-H(\sigma+h)\left(h+\sigma h^{2}\right)^{\frac{3}{2}}-h h^{\prime},
$$

so that we get $h^{\prime \prime}(\alpha)<0$ and we can continue $h$ to an interval $[\alpha-\varepsilon, \alpha]$ in such a way that $h^{\prime}$ is strictly positive on it. Let us denote by $f_{1}$ the inverse function of the restriction of the function $h$ to the interval $[\alpha-\varepsilon, \alpha]: f_{1}$ is then strictly increasing in some interval $[S-\delta, S]$ and $\lim _{s \rightarrow S^{-}} f_{1}^{\prime}(s)=+\infty$. Furthermore, the region bounded by $C$ lies above the graph of $f_{1}$ (since $f_{1}(S-\delta)<f_{1}(S)=$ $=\alpha=f(S)<f(S-\delta))$ and, as a consequence of (5), we have

$$
s f_{1} f_{1}^{\prime \prime}=s f_{1}^{\prime 2}+H\left(s+f_{1}\right)\left(f_{1}+s f_{1}^{\prime 2}\right)^{\frac{3}{2}}-f_{1} f_{1}^{\prime} .
$$

Let us extend the graph of $f_{1}$ to the left as far as possible, until we reach an interval $\left[s_{2}, S\right]$, remaining always in the first quadrant with non negative slope. First of all, we note that it is not possible that $f_{1}\left(s_{2}\right)=0$ : in this case, we have that $f_{1}^{\prime}\left(s_{2}\right)=\frac{1}{h^{\prime}(0)} \geq 0$, contradicting the equation (6) which implies $h^{\prime}(0)<0$. Moreover, it must hold that $s_{2} \neq 0$ : otherwise, the region bounded by $C$ in the first quadrant would be included between the graphs of $f$ and $f_{1}$ and the symmetry of $C$ with respect to both axes would contradict the fact that $D$ is connected and contains the origin. Finally, $f_{1}^{\prime}\left(s_{2}\right)=+\infty$ cannot occur: at the point $f_{1}\left(s_{2}\right)$ the inverse function $h$ would have $h^{\prime}=0$ and (because of (6)) $h^{\prime \prime}<0$; this would contradict the increase of $h$. Therefore, $f_{1}$ can be defined in a maximal interval $\left[s_{2}, S\right]$, so that $f_{1}$ is increasing, the graph of $f_{1}$ is contained in the first quadrant and $f_{1}^{\prime}\left(s_{2}\right)=0$.

Summing up, supposing that the tangent of $C$ becomes vertical at the point $S$, we can follow the graph of $f_{1}$ until a point $s_{2} \in(0, S)$, where the tangent of $C$ becomes horizontal. We may iterate this construction building up a function $f_{2}$ defined in $\left[s_{3}, s_{2}\right]$ : necessarily $s_{3}>0, f_{2}$ is strictly decreasing in the interval $\left(s_{3}, s_{2}\right)$ and $f_{2}^{\prime}\left(s_{3}\right)=-\infty$ (vertical tangent). This process can be continued indefinitely obtaining a spiral contained in $C$, formed by the graphs of functions $f, f_{1}, f_{2}, f_{3}, \ldots$, so that each graph corresponds to a monotonic clockwise turn of $\frac{\pi}{2}$ of the tangent to $C$. Then $C$, seen as a subset of $\mathbb{C}^{2}$, cannot be contained in the boundary of a regular domain.

This contradiction proves that $f(S)=0$. 
We have so proved that $f$ is strictly decreasing in a maximal interval $(0, S)$ and its graph hits the $s$-axis at $S$. Taking $g(s)=\sqrt{f\left(s^{2}\right)}$, the connected component of $C$ containing $(t, 0)$ is properly represented in the first quadrant by the graph of $g$ and the behavior on the other quadrants follows from the symmetry of $C$ with respect to the coordinate axes. The following proposition states that there are no other connected components.

Proposition 2.3. The set $C$ is connected.

Proof. Let us suppose that another connected component of $C$ exists and let us denote it by $C^{\prime}$. The set $C^{\prime}$ cannot contain points such as $(a, 0)$ or $(0, b)$ : we would obtain the same contradiction of the last proof (taking into account that $(a, 0)$ would not be the highest point of this form). On the other hand, if we consider the function $\phi$ which locally defines $C^{\prime}$ (in the same way as $f$ previously), we find that $\phi$ cannot increase or decrease: since $C^{\prime}$ does not intersect the axes, this would cause again the generation of a spiral. Hence, a connected component of $C$ without the point $(t, 0)$ does not exist.

REMARK 2.4. Let us deal with the smoothness of $f$. The $C^{2}$ regularity at $s=0$ was already discussed. The regularity about $s=S$ follows from the regularity of $f^{-1}$ at $s=0$. At a generic point $s_{0} \in(0, S)$ the ODE (2) is not singular, so that the unique solution of the Cauchy problem

$$
\begin{aligned}
f^{\prime \prime} & =\frac{f^{\prime 2}}{f}-\frac{f^{\prime}}{s}-\frac{H(s+f)\left(f+s f^{\prime 2}\right)^{\frac{3}{2}}}{s f} \\
f\left(s_{0}\right) & =a_{0} \\
f^{\prime}\left(s_{0}\right) & =a_{1}
\end{aligned}
$$

is of class $C^{4}$ in a neighborhood of $s_{0}$ (because $H$ is of class $C^{2}$ ).

Summing up, after assuming that $f$ represents $C$ in a neighborhood of $(t, 0)$, we deduced that $f$ belongs to $C^{2}([0, S])$, that determines completely $C$, that satisfies the second order ODE

$$
s f f^{\prime \prime}=s f^{\prime 2}-H(s+f)\left(f+s f^{\prime 2}\right)^{\frac{3}{2}}-f f^{\prime},
$$

and it is such that

$$
f(s)>0 \text { for } 0 \leq s<S, \quad f(S)=0, \quad f^{\prime}(s)<0 \text { for } 0 \leq s<S .
$$

With regard to the ball of radius $R$ centered at the origin, the related function is $f(s)=R^{2}-s$. It satisfies the equation and all the previous properties. 


\section{A second order singular ODE.}

Let us focus our attention on the ordinary differential equation (2). It is a second order ODE which is singular at $s=0$. We begin with a local existence and uniqueness result.

Proposition 3.1. Let $H$ be a locally Lipschitz-continuous function defined in an open interval I. Let $a \in I$ be a strictly positive number. Then, there exist $\delta>0$ and a unique solution $f \in C^{1}([0, \delta]) \cap C^{2}(] 0, \delta[)$ satisfying the equation

$$
s f f^{\prime \prime}=s f^{\prime 2}-H(s+f)\left(f+s f^{\prime 2}\right)^{\frac{3}{2}}-f f^{\prime},
$$

and such that $f(0)=a$.

Proof. Since $f(0)>0$, the differential equation may be rewritten as

$$
\left(s f^{\prime}\right)^{\prime}=f^{\prime}+s f^{\prime \prime}=\frac{s f^{\prime 2}}{f}-\frac{H(s+f)\left(f+s f^{\prime 2}\right)^{\frac{3}{2}}}{f}=G\left(s, f, s f^{\prime 2}\right),
$$

where $G(t, x, y)=\frac{y}{x}-\frac{H(t+x)(x+y)^{\frac{3}{2}}}{x}$ is locally Lipschitz-continuous in the open set $\Omega=\left\{(t, x, y) \in \mathbb{R}^{3}: x>0, x+y>0, t+x \in I\right\}$. We thus reduce the problem to that of demonstrating existence and uniqueness of a function $f \in C^{1}([0, \delta]$ ) (for some $\delta>0$ ) satisfying

$$
f^{\prime}=\frac{1}{s} \int_{0}^{s} G\left(\sigma, f(\sigma), \sigma f^{\prime 2}(\sigma)\right) d \sigma,
$$

and such that $f(0)=a$. Because of the continuity of $G$, we have that $s f^{\prime} \in C^{1}$, so that $f$ is of class $C^{2}$ away from $s=0$.

To proceed with the proof, we can argue in analogy with the classical proof of the Cauchy-Lipschitz's theorem. Let us consider the operator $T$ defined by

$$
T u(s)=\frac{1}{s} \int_{0}^{s} G\left(\sigma, a+\int_{0}^{\sigma} u(t) d t, \sigma u^{2}(\sigma)\right) d \sigma,
$$

where $u$ is a continuous function such that the right-hand side is well defined. It is not difficult to show that for every $M$ enough large there exists $\delta_{M}>0$ such that, if we define $Y=Y_{M, \delta}=\left\{u \in C([0, \delta]): \sup _{[0, \delta]}|u| \leq M\right\}$ for $0<\delta<\delta_{M}$, we get $T: Y \longrightarrow Y$ and $T$ is a contraction on the Banach space 
$Y$. By the Banach's theorem, there exists only one continuous function $u$ defined in $[0, \delta]$ (for some $\delta>0$ ) which is a fixed point for the operator $T$. Hence, if we set

$$
f(s)=a+\int_{0}^{s} u(t) d t,
$$

for $s \in[0, \delta]$, we get that $f$ is the unique function belonging to $C^{1}([0, \delta])$ such that $f(0)=a$ and satisfying the integral equation (7).

CoRollary 3.2. Let $f, \tilde{f}:[0, S] \rightarrow \mathbb{R}$ be two solutions of class $C^{2}$ of the equation (2), such that they are strictly positive in [0,S[. Let us assume that $f(0)=\tilde{f}(0)>0$. Then $f=\tilde{f}$.

Proof. By Proposition 3.1, $f=\tilde{f}$ in an interval $[0, \delta]$, for a suitable $\delta>0$. On the other hand, for $s \neq 0$ the equation (2) is non-singular (provided that the solution does not vanish). Then, for the uniqueness of the solution of a Cauchy problem, we have $f=\tilde{f}$ in $[0, S[$, so that, by continuity, up to $s=S$.

\section{A uniqueness result: a sphere theorem.}

Let $f$ be the function of Section 2. Here we show that the equation (2) determines some initial conditions for $f$. This fact, together with the result of Section 3, will give us a uniqueness result.

To this end, we will use the following crucial result proved in [5].

Theorem (Comparison Principle) 4.1. Let $D_{1}$ and $D_{2}$ be strictly Levi-pseudoconvex domains of $\mathrm{C}^{2}$ with connected boundaries. Let us assume that $D_{1} \subseteq D_{2}$ and $\partial D_{1} \cap \partial D_{2} \neq \emptyset$. Moreover, we suppose that the Levi curvature of $D_{2}$ is greater than or equal to the Levi curvature of $D_{1}$. Then $D_{1}=D_{2}$.

Firstly, we show that $f(0)$ determines $f^{\prime}(0)$ and $f^{\prime \prime}(0)$.

LEMMA 4.2. The first and second derivatives of $f$ at $s=0$ depend on $f(0)$ in the following way:

$$
\begin{gathered}
f^{\prime}(0)=-H(f(0)) \sqrt{f(0)} \\
f^{\prime \prime}(0)=\frac{1}{2}(1-H(f(0)) \sqrt{f(0)})\left(\frac{3}{2} H^{2}(f(0))-H^{\prime}(f(0)) \sqrt{f(0)}\right) .
\end{gathered}
$$


Proof. The identity concerning $f^{\prime}(0)$ simply follows from putting $s=0$ in equation (2). With regard to the second one, we note that, for $0<s<S$, we have

$$
f^{\prime \prime}=\frac{f^{\prime 2}}{f}-\frac{f f^{\prime}+H(s+f)\left(f+s f^{\prime 2}\right)^{\frac{3}{2}}}{s f} .
$$

As $s \rightarrow 0^{+}$, we can apply L'Hôpital's rule to the second term in the righthand side, since the numerator vanishes at $s=0$ for the first identity. Then, at $s=0$, we get

$$
f^{\prime \prime}=\frac{f^{\prime 2}}{f}-\frac{1}{f}\left(f^{\prime 2}+f f^{\prime \prime}+H^{\prime}(f)\left(1+f^{\prime}\right) f^{\frac{3}{2}}+\frac{3}{2} H(f) f^{\frac{1}{2}}\left(f^{\prime}+f^{\prime 2}\right)\right),
$$

from which we obtain

$$
\begin{aligned}
2 f^{\prime \prime}(0) & =-\frac{1+f^{\prime}(0)}{\sqrt{f(0)}}\left(H^{\prime}(f(0)) f(0)+\frac{3}{2} H(f(0)) f^{\prime}(0)\right) \\
& =(1-H(f(0)) \sqrt{f(0)})\left(\frac{3}{2} H^{2}(f(0))-H^{\prime}(f(0)) \sqrt{f(0)}\right) .
\end{aligned}
$$

Before proceeding, let us suppose that the Levi curvature of $\partial D$ is nonincreasing as a function of the distance from the origin, i.e. we assume the condition

$$
H^{\prime} \leq 0 \text {. }
$$

We can now prove the following proposition.

Proposition 4.3. The function $f$ satisfies

$$
H(f(0)) \sqrt{f(0)}=1,
$$

or equivalently $f^{\prime}(0)=-1\left(\right.$ or $\left.f^{\prime \prime}(0)=0\right)$.

Proof. Let us argue by contradiction. We first suppose $H(f(0)) \sqrt{f(0)}>1$. Then, by the previous lemma and the hypothesis (8) on the function $H$, we get $f^{\prime}(0)<-1$ and $f^{\prime \prime}(0)<0$. Let us define

$$
s_{0}=\max \left\{s \in[0, S]: f^{\prime \prime}(\sigma) \leq 0 \text { for } 0 \leq \sigma \leq s\right\},
$$

and put

$$
\lambda:[0, S] \rightarrow \mathbb{R}, \quad \lambda(s)=f\left(s_{0}\right)+f^{\prime}\left(s_{0}\right)\left(s-s_{0}\right)=\alpha-\beta s .
$$


Since $f$ is concave in $\left[0, s_{0}\right]$, we have

$$
\beta=-f^{\prime}\left(s_{0}\right) \geq-f^{\prime}(0)>1, \quad \text { and } \quad \lambda(s) \geq f(s) \text { for } 0 \leq s \leq s_{0} .
$$

In particular, $\alpha=\lambda(0) \geq f(0)>0$. Let us now consider the function

$$
g:[0, S] \rightarrow \mathbb{R}, \quad g(s)= \begin{cases}\lambda(s), & \text { se } 0 \leq s \leq s_{0} \\ f(s), & \text { se } s_{0} \leq s \leq S\end{cases}
$$

The function $g$ is of class $C^{2}$ and $g(s) \geq f(s)$ for all $s \in[0, S]$. Therefore,

$D_{f}:=\left\{\left(z_{1}, z_{2}\right) \in \mathbb{C}^{2}:\left|z_{1}\right|^{2}<f\left(\left|z_{2}\right|^{2}\right\} \subseteq D_{g}:=\left\{\left(z_{1}, z_{2}\right) \in \mathbb{C}^{2}:\left|z_{1}\right|^{2}<g\left(\left|z_{2}\right|^{2}\right)\right\}\right.$.

For $z=\left(z_{1}, z_{2}\right) \in \partial D_{g}$ and $s=\left|z_{2}\right|^{2}$, we define

$$
K(s)=K_{z}\left(\partial D_{g}\right) .
$$

By the formula (4), for $0 \leq s \leq s_{0}$, we obtain

$$
K(s)=\frac{\alpha \beta}{(\alpha+\beta s(\beta-1))^{\frac{3}{2}}} .
$$

Since $\beta>1, K$ is strictly decreasing in $\left[0, s_{0}\right]$, so that

$$
K(s) \geq K\left(s_{0}\right)=H\left(s_{0}+f\left(s_{0}\right)\right) \text { for } 0 \leq s \leq s_{0} .
$$

If $s_{0}<S$, the last equality follows from the fact that $f$ and $g$ are equal up to the second derivative at $s_{0}$. If $s_{0}=S, f$ and $g$ are equal at $s_{0}$ only up to the first derivative, but they vanish at $s_{0}$, so that the curvature does not depend on the second derivative. However, the function $s \mapsto H(s+f(s))$ has first derivative $H^{\prime}(s+f(s))\left(1+f^{\prime}(s)\right)$ and it is increasing in $\left[0, s_{0}\right]$. Therefore,

$$
K(s) \geq H(s+f(s)),
$$

for all $s \in[0, S]$. Then, we may invoke the Comparison Principle and state that $D_{f}=D_{g}$. Hence, $f=g$ and $K(s)=H(s+f(s))$ for all $s \in[0, S]$. Since $K$ is strictly decreasing and $H(s+f(s))$ is increasing in the interval [0, $\left.s_{0}\right]$, it is a contradiction. This prove that $H(f(0)) \sqrt{f(0)} \leq 1$.

On the other hand, if we argue as above we reach a contradiction also assuming $H(f(0)) \sqrt{f(0)}<1$.

We close this section by giving the proof of our main theorem.

Proof of Theorem 1.1. In Section 2, we showed that $\partial D$ can be represented by a function $f$ satisfying

$$
s f f^{\prime \prime}=s f^{\prime 2}-H(s+f)\left(f+s f^{\prime 2}\right)^{\frac{3}{2}}-f f^{\prime} .
$$


In Proposition 4.3, we proved that the initial value $f(0)=a$ must satisfy $H(a) \sqrt{a}=1$. By inserting the function $f(s)=a-s$ in (2), we get

$$
0=s-H(a) a^{\frac{3}{2}}+a-s=a(1-H(a) \sqrt{a}) .
$$

By Corollary 3.2, it follows that $f(s)=a-s$ is the unique solution such that $f(0)=a$. Therefore, the set $C$ is completely determinated by the equation $x_{1}^{2}=a-x_{2}^{2}$, which represents a circle of radius $\sqrt{a}$. Hence, $\partial D$ is a sphere of radius $\sqrt{a}$ centered at the origin.

Acknowledgments. We are deeply indebted to Ermanno Lanconelli for several helpful discussions about this topic and about the approach to the mathematics.

\section{REFERENCES}

[1] J. G. Hounie - E. Lanconelli, An Alexandrov type theorem for Reinhardt domains of $\mathbb{C}^{2}$, Contemporary Math., 400 (2006), pp. 129-146.

[2] J. G. Hounie - E. LANCONELli, A sphere theorem for a class of Reinhardt domains with constant Levi curvature, Forum Math., 20 (2008), pp. 571-586.

[3] S. G. KRANTZ, Function theory of several complex variables, Wiley, New York, 1982.

[4] V. MARTINo - A. MontANARI, Integral formulas for a class of curvature PDE's and applications to isoperimetric inequalities and to symmetry problems, Forum Math, 22 (2010), pp. 255-267.

[5] A. Montanari - E. Lanconelli, Pseudoconvex fully nonlinear partial differential operators: strong comparison theorems, J. Differential Equations, 202 (2004), pp. 306-331.

[6] R. Monti - D. Morbidelli, Levi umbilical surfaces in complex space, J. Reine Angew. Math., 603 (2007), pp. 113-131.

Manoscritto pervenuto in redazione il 26 aprile 2010. 